\title{
Author/Subject Indexes Vol. 158, No. 1, 1997
}

Antinoff,N. 54 Bérubé, S. 26 Bore, P. 30 Boyes,R. 30 Cook, P. 33 DeNovo,R.C. 48 Doran,G.A. 37 Dower, R. 30 Eckel, H.E. 74 Entius, C.A.C. 44 Graf,J. 68 Grondin, G. 26 Henry, R.W. 26, 48, 54 Holstege, J.C. 44 Jakob, D.S. 68 Janick, L. 26, 48, 54 Kekic,M. 37 Koike, F. 21 Magiros, M. 37

Masuda,Y. 10 Matsumura, A. 59 Minagawa, K. 10 Orosz, S. 54 Ricke, S. 74 Rijn, R.R. van 44 Saito,K. 59 Schiltenwolf, M. 68 Shimaguchi, S. 21 Sittel,C. 74 Stennert,E. 74 Stoeckart, R. 44 Taguchi, M. 21 Weiglein, A.H. 5,6 Yohro,T. 10 Yoshida,Y. 10,21 Zhang, J. 10 Zwamborn, A.W. 44

Subject Index Vol. 158, No. 1,1997

Acetone reclamation 26

Anatomy 26

-, sectional 33, 37, 44, 54, 68, 74

Birds 54

Computed tomography 44, 54

Database 10

Endoscopic technique 48

Explosive vapours 30

Extensor 59

Foot 59

Infraorbital sinus 54

Internet 10

Intraosseous injection 68

Larynx 74

Lifting gear 30

Lunate bone necrosis 68

Macaque 59

Magnetic resonance imaging 37, 44

Morphometry 74

Muscle fiber composition 59

- spindle 59

Neuroanatomy 6

Neuroscience 6

Parallax panoramagram 21

Paranasal sinus 54

Plastination 6, 10, 21, 26, 30, 33, 37, 44,

48,54,68,74 -, epoxy 33, 44, 54, 59, 68, 74 -, epoxy resin and gelatin 59 -, PEM11 37 -, polyester 6 
-, sheet 33, 44, 54, 59, 68, 74 -, silicone 6, 48 Preservation 33 Psittacine 54 Quantitative analysis 59 Sheet plastination 44, 68 Stereograph 21 Submacroscopy 33 Teaching 6, 26, 33, 44, 48

Venous congestion 68 Volumegraph 21 Whole-organ histology 74

(C) 1997 S. KargerAG, Basel

E-Mail karger@karger.ch Fax + 41613061234 http://www. karger. ch 